\title{
Spinning of hollow fiber ultrafiltration membranes from a polymer blend
}

\author{
I.M. Wienk ${ }^{1}$, F.H.A. Olde Scholtenhuis, Th. van den Boomgaard * , C.A. Smolders \\ University of Twente, P.O. Box 2177500 AE Enschede, Netherlands
}

Received 12 October 1993; revised 27 March 1995; accepted 3 April 1995

\begin{abstract}
In this study the dry-wet spinning technique is used for the preparation of hollow fiber membranes. In the polymer solution a blend of two polymers, poly (ether sulfone) and poly (vinyl pyrrolidone), is used. The morphology of the membranes obtained is related to rheological characteristics and phase behavior of the polymer solution during spinning. The outer surface pore structure is mainly dependent on the conditions in the airgap. The typical performance of the membranes lies in the ultrafiltration region.
\end{abstract}

Keywords: Membrane formation; Polymer blend; Spinning; Ultrafiltration

\section{Introduction}

The spinning of hollow fibers is very often applied as a preparation technique for ultrafiltration membranes. Even so the number of fundamental contributions to this subject is limited [1-6]. Much knowledge about the spinning of porous hollow fiber membranes has been described in patents. In the spinning process both phase separation and rheological phenomena will have an influence on the shape and morphology of the membrane fibers.

In this study membranes are made from a blend of two polymers. Poly (ether sulfone) (PES) is used as membrane forming polymer. The thermal stability is very high $\left(190^{\circ} \mathrm{C}\right.$ for continuous use) which makes the membranes resistant to steam sterilization. PES is also stable with respect to thermal oxidation [7], and resis-

\footnotetext{
* Corresponding author.

${ }^{1}$ Present adress: NPBI, P.O. Box 30, 7880 AA Emmer Compascuum, Netherlands.
}

tant to the attack of mineral acids, alkali and salt solutions. Moreover the resistance of PES to detergents and hydrocarbons is good [8].

The hydrophilic polymer poly (vinyl pyrrolidone) (PVP) is used as an additive in the polymer solution. The introduction of this polymer will give the membranes a hydrophilic character. It is known $[9,10]$ that hydrophilic materials are less susceptible to adsorption of proteins, which for industrial applications of membranes is a very attractive behavior. PVP is a well known additive in membrane forming systems [1$5,11-14]$. The effects of this additive are an increase in viscosity of the polymer solution, an influence on macrovoid formation and an increase in permeability of the membranes. Other hydrophilic polymers like poly (ethylene oxide) have similar effects on membranes as described here for PVP.

The membranes described here are made using the dry-wet spinning technique. A number of spinning parameters has been varied. Special attention is paid to the 
conditions in the airgap. Here the amount of water vapor diffusing into the polymer solution can be varied either by changing the humidity of the airgap or by varying the residence time of the nascent membrane in the airgap.

The influence of the spinning parameters on the morphology and the performance of the membranes is discussed in light of existing theories on membrane formation.

\section{Theory}

\subsection{Membrane formation by diffusion induced phase separation}

Phase separation of a polymer solution can be induced by:

-quenching the solution to a lower temperature

-immersion of the solution in a bath of non-solvent

-contacting the polymer solution with a vapor of the non-solvent

-evaporation of the solvent.

For membrane formation, immersion precipitation is the most important procedure. Phase separation processes by evaporation or contact with a non-solvent vapor are closely related to the immersion precipitation process because they are also diffusion induced.

Fundamental studies on membrane formation by immersion precipitation in ternary systems of polymer, solvent and non-solvent have been carried out by Cohen et al. [15] Reuvers et al. [16], Tsay et al. [17] and Radovanovic et al. [18]. They developed mass transfer models to describe the diffusion processes especially for the first moments of immersion.

The mass transfer models are valid only for homogeneous polymer solutions. As soon as the polymer solution demixes the mass exchange with the immersion bath will be different for the two phases. More information, than is available now, is necessary on how the compositions and the volumes of the two phases change in time to be able to expand the mass transfer model for the inhomogeneous region.

It is important to note that the thermodynamic data of the binodal and spinodal curves are based on equilibrium conditions. If the kinetics of the process are very rapid the equilibrium thermodynamics will not give adequate information on the compositions in the demixing system at the time and place of inspection. This will be important especially in the formation of top layers of ultrafiltration membranes because here the diffusion rates of solvent and non-solvent are usually very high. The polymer being a macromolecular component with a relatively small diffusion coefficient is not able to respond to the fast diffusion processes of solvent and non-solvent. How this will result in a nodular structure which is a typical structure for the top layers of ultrafiltration membranes is dealt with in related publications $[19,20]$.

Despite its restrictions the mass transfer model is suitable to explain qualitatively the effects of system variables, such as concentrations. temperature and interaction between the components, on the structure of the ultimate membrane.

\subsection{Influence of PVP on the membrane formation process}

When PVP is added to the polymer solution the structure of the membranes changes drastically as was mentioned in the introduction. Roesink [5] ascribed the high interconnectivity of the pores to a thin PVP layer present in between the pores. This layer is formed during phase separation and can easily break up upon drying.

For the quaternary system PES/PVP/NMP/water Boom et al. [21-24] followed a different approach of phase separation. Two timescales for diffusion are defined.

One is a short timescale for the diffusion of solvent and non-solvent. On this scale the interdiffusion between the two polymers is negligible. The two polymers act as one and the system is quasi ternary. The binodals belonging to this ternary system are called virtual binodals. For the quasi ternary system the demixing gap is very small.

On the other hand there is a long timescale for the diffusion of the two polymers with respect to each other. The binodal belonging to the quaternary system is indicated as the equilibrium binodal. This binodal coincides with the cloudpoint curve of the system. For the first moments (fractions of seconds) after immersion the phenomena taking place on a short time scale are the most important events. But as the diffusion processes proceed further the long timescale becomes more important. 
Boom et al. [23,24] reason that for these systems spinodal decomposition occurs which results in high interconnectivity of the pores. Spinodal demixing is characterized by a structure in which both phases are continuous (co-continuous structure). The interconnected pores are the continuous polymer lean phase intertwined by the continuous polymer rich phase which forms the membrane matrix.

\subsection{Membrane formation by spinning hollow fibers from the system PES/PVP/NMP/water}

In the dry-wet spinning technique there are three stages of diffusion induced phase separation:

-vapor penetration of non-solvent at the outer surface in the airgap

-immersion precipitation at the outer surface, once the nascent membrane has passed the airgap and enters the water bath

-immersion precipitation from the inside (through diffusional exchange with a bore liquid)

Besides this, the polymer solution can also undergo a temperature quench if the temperature of the coagulation medium is lower than the extrusion temperature of the polymer solution and below the thermal phase separation temperature.

In the airgap between spinneret and coagulation bath a vapor of the non-solvent water is supplied. The water diffuses into the extruded polymer solution. Out-diffusion (evaporation) of solvent can be neglected because the temperature in the airgap is far below the boiling temperature of the solvent. If the initial composition of the polymer solution is already close to the cloudpoint composition, the indiffusion of water vapor can induce phase separation in the airgap. Since the indiffusion of water from the vapor phase is not very fast and the outdiffusion of solvent is nil the difference between the short and long time scale for demixing is not so profound. The supersaturation will therefore not be large. An increase of the water vapor concentration in the airgap will result in an increased driving force and a higher water concentration in the outer layer of the polymer solution. Upon phase separation of this polymer solution the volume of the polymer lean phase will be larger.

Vitrification of the polymer rich phase in the airgap is not likely to occur because the residence time is too short for the solvent to diffuse to the polymer lean phase and the solvent cannot diffuse out of the solution. The gelation will therefore take place during immersion in the coagulation bath. In this bath non-solvent diffuses into the polymer casting solution while solvent diffuses out. In the system of study the coagulation strength of the non-solvent is high. The strength is high because of a high interaction of the non-solvent with the solvent and a low interaction between non-solvent and the polymer (PES). The diffusion processes of solvent and non-solvent will be fast due to their high mutual interaction. In this situation the existence of the short timescale (for solvent and non-solvent) and the long timescale (for the two polymers) becomes important and may result in a high supersaturation of the polymer solution.

In the top layer of the nascent membrane where demixing already starts in the airgap, the diffusion processes in the water bath rapidly lead to vitrification of the polymer (PES) rich phase. The pore volume of this layer is mainly determined by two processes: (i) the amount of water that diffuses into the solution during its contact time with the vapor and (ii) the amount of solvent that diffuses into the coagulation bath. Below the top layer the phase separation starts in the coagulation bath. Here the large supersaturation can lead to spinodal demixing of the polymer solution and thus the formation of a co-continuous membrane structure. The polymer concentration in the sublayer will not be increased as much as in the top layer because of less outdiffusion of solvent. The pores in the sublayer will therefore be larger the more remote they are from the surface.

At the bore side the coagulation strength of the bore liquid, starting from the pure non-solvent, generally is reduced by the addition of solvent. Diffusion processes of solvent and non-solvent are slower then, since the driving forces (concentration gradients) are lower. Especially the out-diffusion of solvent is retarded. The result is a relatively low polymer concentration during phase separation and a high porosity. Normally such coagulation media would give a delay time for demixing. However in this case the polymer solution will demix instantaneously because the presence of PVP causes a shift of the virtual binodal towards the equilibrium binodal.

In the spinning process the diffusion processes are combined with a temperature effect. The extrusion temperature of the polymer solution is often higher than 
the temperature of the coagulation medium. The temperature can affect the phase separation process in two ways. Reducing the temperature may (i) induce demixing of the polymer solution and (ii) reduces the diffusion coefficients of the components. The growth of the nuclei therefore is slowed down and gelation of the polymer rich phase is promoted. The general effect is that at a lower temperature the velocity of the phase separation process is slowed down whereas the vitrification point is reached at a lower polymer concentration.

Besides phase separation rheological phenomena may influence the structure of the membrane in the spinning process. Aptel et al. [ 1] have spun fibers from a solution containing polysulfone, PVP and DMAc with the skin on the inside. The bore liquid used was water, causing fast precipitation of the polymer. They found that the performance of the membranes depends on the extrusion rates of the polymer solution and on the bore liquid. According to Aptel shear forces in the spinneret cause orientation of polymer molecules. If gelation of the solution due to contact with the bore liquid is faster than the relaxation time of the polymer solution the stretched polymer chains are frozen in and the permeability of the membrane is lower. Roesink [5] mentioned that the shear forces will lead to a dieswell of the polymer solution after it leaves the spinneret. Any effect on the performance of the membranes was not reported. The diameter of the hollow fiber depends on the take-off rate and on the extrusion rate of the polymer solution as was published by Cabasso et al. [3]. Espenan et al. [2] found that the diameter of the fiber decreases when the airgap becomes longer or when the flow rate of the polymer solution decreases. The diameter of the bore increased if the flow rate of the bore liquid was enhanced. An empirical relation was found between the wall thickness and the permeability but it was not based on a physical model.

Finally, the performance of the ultimate membranes is effected by post-treatment of the fibers such as glycerination and drying steps. Research on the post-treatment of PES/PVP fibers is beyond the scope of this article. Interested readers are referred to the literature [25].

\section{Materials and methods}

\subsection{Materials}

Poly (ether sulfone) (PES) was purchased from ICI (Victrex 5200P. $M_{\mathrm{w}} 44000 \mathrm{~g} / \mathrm{mol}$ ). Later on spinning experiments with a controlled humidity in the airgap were performed using PES from BASF (Ultrason E6010P). The weight average molecular weight of this polymer was somewhat lower $(43000 \mathrm{~g} / \mathrm{mol})$. Two types of poly ( vinyl pyrrolidone) (PVP) from Janssen Chimica were used, $\mathrm{K} 90\left(M_{\mathrm{w}} 507000 \mathrm{~g} / \mathrm{mol}\right)$ and $\mathrm{K} 30$

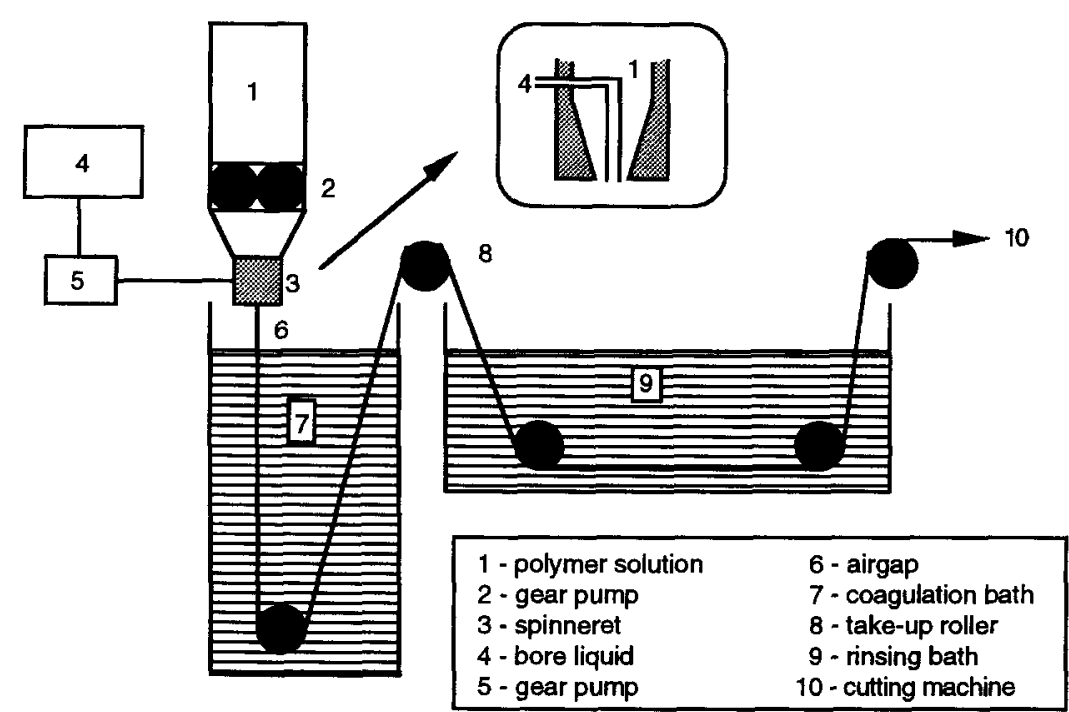

Fig. 1. Spinning set-up with transport of the fibers to a rinsing bath. Conditions of the airgap are dependent on the water bath below the airgap. 
$\left(M_{\mathrm{w}} 18000 \mathrm{~g} / \mathrm{mol}\right)$. The weight average molecular weights of the polymers were determined using GPC. The polymers were used as received. The water content of the polymers was measured in a Buchi oven and was $1.5 \mathrm{wt} \%$ for PES and $6 \mathrm{wt} \%$ for PVP. The solvent 1 methyl-2-pyrrolidone (NMP) was purchased from Merck ( synthetic grade). The non-solvent was water.

\subsection{First spinning set-up}

The first set-up is shown in Fig. 1. The polymer solution was contained in a thermostated vessel. With a gear pump it was extruded through a tube-in-orifice spinneret. For the bore liquid also a gear pump was used and it was pumped through the inner orifice of the spinneret. The dimensions of the spinneret were 0.8 $\mathrm{mm}$ for the outer diameter of the inner orifice and 1.5 $\mathrm{mm}$ for the diameter of the outer orifice. The thickness of the needle wall was $0.1 \mathrm{~mm}$. The airgap is the space between the spinneret and the coagulation bath. The humidity and temperature of the airgap were determined by the temperature of the water bath below the airgap. Once the fiber was coagulated in the water bath it could be transported by rollers. The fibers were cut automatically in pieces of about $30 \mathrm{~cm}$. The fibers were collected in a rinsing bath. By cutting the fibers into shorter lengths the bore liquid could be flushed out more readily.

\subsection{Second spinning set-up}

To be able to control the humidity and temperature of the airgap irrespective of the conditions of the coagulation bath in the second set-up a casing was placed between the spinneret and the water bath. In the casing the airgap was $5 \mathrm{~cm}$ long. A nitrogen stream was saturated with water of $60^{\circ} \mathrm{C}$ and mixed with a heated but dry nitrogen stream. The mixed gas was lead to the space around the fiber and then slowly flows upwards with a linear velocity of $2.75 \mathrm{~cm} / \mathrm{s}$. The temperature in the isolated casing was $50^{\circ} \mathrm{C}$. A schematic representation of this set-up is shown in Fig. 2.

The humidity of the saturated nitrogen stream can be calculated using the Antoine equation for saturated vapor pressures:

$\log P_{\mathrm{i}}^{0}=A+\frac{B}{(C+T)}$

with $P_{\mathrm{i}}^{0}$ the partial vapor pressure at saturation (in $\mathrm{mmHg}$ ) and temperature $T$ (in ${ }^{\circ} \mathrm{C}$ ). The Antoine constants for water are [26]: $A=8.071 ; B=-1730.630$; $C=233.426$.

The humidity of the airgap is found by taking the volume average of the dry and saturated nitrogen stream. It can be varied between the partial pressure of the coagulation bath below the airgap (water bath of $20^{\circ} \mathrm{C}, P_{\mathrm{H}_{2} \mathrm{O}}^{\mathrm{O}}=0.023 \mathrm{Bar}$ ) and the temperature in the

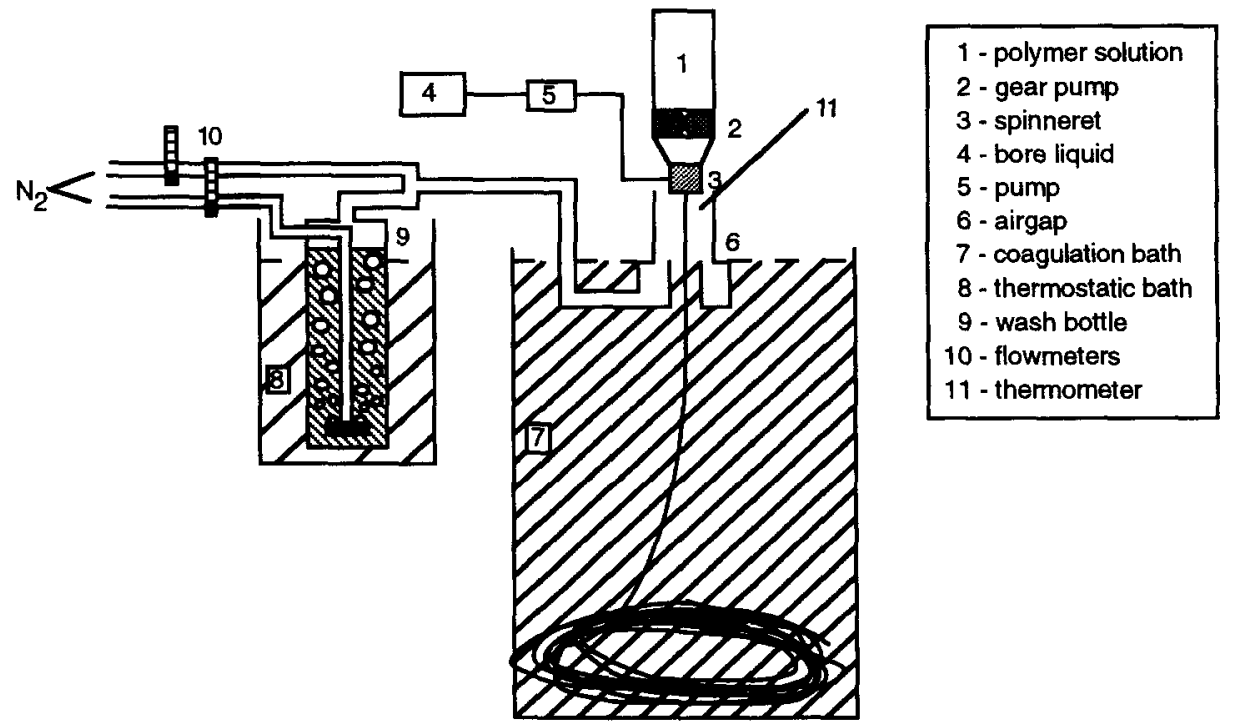

Fig. 2. Spinning set-up with controlled humidity in the airgap. Fibers are collected at the bottom of the coagulation bath. 
airgap which is $50^{\circ} \mathrm{C}\left(P_{\mathrm{H}_{2} \mathrm{O}}^{0}=0.123 \mathrm{Bar}\right)$. If the partial pressure would become higher water vapor would condense on the wall of the casing and on the fiber.

\subsection{The spinning process and post-treatment}

Clear polymer solutions were obtained by dissolving (at $80^{\circ} \mathrm{C}$ ) PES and PVP in NMP and adding water to a known amount. All polymer spinning solutions contained $20 \mathrm{wt} \%$ PES and $5 \mathrm{wt} \%$ water. The concentration and molecular weight of the PVP was varied. The viscosity of the polymer solutions was measured using a rotary viscometer (Brabender). Unless mentioned otherwise the bore liquid contained $78 \mathrm{wt} \% \mathrm{NMP}$ and 3 wt\% PVP in water.

The polymer solutions and bore liquids were filtered by a metal filter $(25 \mu \mathrm{m})$ and were degassed before use. Temperature of the bore liquids was $25^{\circ} \mathrm{C}$. After spinning and coagulation the fibers were rinsed with water of $50^{\circ} \mathrm{C}$ for 2 days. Following earlier work in our laboratory (Roesink [5]), excess PVP could be removed after contacting the fibers with a $4000 \mathrm{ppm}$ sodium hypochlorite solution for $48 \mathrm{~h}$. After rinsing for 1 more day the fibers were put in a water bath containing $10 \mathrm{wt} \%$ glycerol for 1 day and finally they were dried at room temperature.

\subsection{Characterization methods}

Membrane morphology was studied using a scanning electron microscope (Jeol, JSM T220A). Water for flux measurements was prefiltered by reverse osmosis. BSA retentions were measured using a $0.1 \mathrm{wt} \%$ BSA solution. BSA $\left(M_{\mathrm{w}} 65000 \mathrm{~g} / \mathrm{mol}\right)$ was pur- chased from Sigma Chemical company and dissolved in a phosphate buffer solution of $\mathrm{pH} 7.4$, containing 0.1 $\mathrm{M} \mathrm{NaCl}$. Flux and retention measurements were carried out in a cross-flow filtration setup. The applied pressure difference was 2 bars and after 30 min conditioning time the water flux was practically constant. BSA concentrations were determined spectrophotometrically at $280 \mathrm{~nm}$. The retention $(R)$ was calculated according to the formula:

$R=1-C_{\mathrm{p}} / C_{\mathrm{f}}(\times 100 \%)$

in which $C_{\mathrm{p}}$ and $C_{\mathrm{f}}$ represent the BSA concentration in the permeate and in the feed, respectively.

\section{Results and discussion}

\subsection{Dry-wet spinning technique}

Fibers were spun using the spinning set-up as depicted in Fig. 1 (uncontrolled conditions in the airgap). The spinning solution contained $20 \mathrm{wt} \%$ PES, 5 wt $\%$ PVP K30, 5 wt \% PVP K 90 and 5 wt $\%$ water. The length of the airgap has been varied for two temperatures of the water bath. The humidity and temperature of the airgap are related to the temperature of the water bath underneath. The residence time of the nascent membrane in the airgap depends on the length of the airgap and the spinning velocity. Airgap residence times are roughly between 0.1 and $0.5 \mathrm{~s}$. The spinning velocity is not an independent variable because in the airgap stretching of the spinning solution occurs due to gravity force. The influence of the gravity force on the nascent fiber is more profound as the length of the

Table 1

Spinning conditions and diameters of the fibers. The composition of the polymer casting solution was 20 wt $\%$ PES, 5 wt\% PVP K30, 5 wt\% PVP K 90 and $5 \mathrm{wt} \%$ water in NMP. The polymer solution was kept at $50^{\circ} \mathrm{C}$. The bore liquid consisted of $78 \%$ NMP and 3 wt $\%$ PVP in water. The temperature of the bore liquid was $25^{\circ} \mathrm{C}$, its flow rate was $2.6 \mathrm{ml} / \mathrm{min}$. The viscosity at zero shear force is 75800 cpoise at $22^{\circ} \mathrm{C}$ and 25400 cpoise at $44^{\circ} \mathrm{C}$

\begin{tabular}{llllll}
\hline $\begin{array}{l}\text { Temperature water bath } \\
\left({ }^{\circ} \mathrm{C}\right)\end{array}$ & $\begin{array}{l}\text { Length airgap } \\
(\mathrm{cm})\end{array}$ & $\begin{array}{l}\text { Extrusion rate } \\
(\mathrm{ml} / \mathrm{min})\end{array}$ & $\begin{array}{l}\text { Take-up speed } \\
(\mathrm{m} / \mathrm{min})\end{array}$ & $\begin{array}{l}\text { External diameter } \\
(\mathrm{mm})\end{array}$ & $\begin{array}{l}\text { Internal diameter } \\
(\mathrm{mm})\end{array}$ \\
\hline 22 & 1 & 4.5 & 4.2 & 1.40 & 0.88 \\
22 & 3 & 4.5 & 5.6 & 1.21 & 0.75 \\
22 & 5 & 5.3 & 7.4 & 1.16 & 0.74 \\
44 & 1 & 4.5 & 4.1 & 1.17 & 0.88 \\
44 & 3 & 4.5 & 5.9 & 1.10 & 0.74 \\
44 & 5 & 5.3 & 7.8 & 0.65 \\
\hline
\end{tabular}



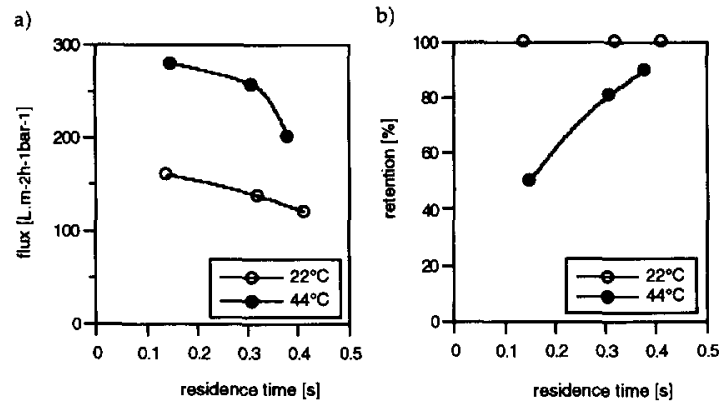

Fig. 3. Performance of the membranes spun with the conditions mentioned in Table 2: (a) water flux and (b) retention for a $0.1 \mathrm{wt} \%$ BSA solution. Residence time in the airgap is the ratio of the length of the airgap and the take-up speed. Deviations from mean values are for water fluxes within $10 \%$ and for retentions within $2 \%$.

airgap is larger and as the viscosity of the polymer solution is lower. In Table 1 it is shown that if the length of the airgap is larger, the take-up speed of the fiber has to be higher to keep up with the extruded fiber. Also the ultimate diameter of the fiber decreases when the length of the airgap increases. Besides gravity force also die-swell and shear forces during extrusion are effective on the polymer solution.

At $44^{\circ} \mathrm{C}$ the effects of fiber stretching were stronger. Compared to $22^{\circ} \mathrm{C}$ the take-up speed increased even more going from an airgap length of 1 to $5 \mathrm{~cm}$. Also for the same length of the airgap the dimensions of the fibers were smaller at $44^{\circ} \mathrm{C}$. The reason for this is that the viscosity of the polymer casting solution is lower at a higher temperature hence gravity force has more impact. The polymer spinning solution which is kept at $50^{\circ} \mathrm{C}$ in the container will cool down rapidly until it reaches the temperature of the airgap.

After post-treatment of the membrane fibers water flux and retention for BSA have been measured. In Fig. 3 water flux and retention are plotted versus the residence time which is the ratio of the length of the airgap and the take-up speed. If the residence time in the airgap went up water flux decreased while retention increased. At a higher temperature of the water bath membranes were made with a higher water flux and a lower retention for $\mathrm{BSA}$ both being more dependent on the residence time in the airgap than at $22^{\circ} \mathrm{C}$.

These results are not consistent with the theory of membrane formation by phase separation. According to this theory a longer contact time with water vapor will allow more water to diffuse into the polymer solution while outdiffusion of non-solvent is not possible.
A more open porous structure would be expected, resulting in a high water flux through the membrane. The observed low water flux at longer residence time leads to the conclusion that gravity force on the nascent fiber and fiber stretching influence the membrane formation in such a way that it hinders the generation and growth of pores. A physical model to explain these phenomena is not easy to derive since apart from gravity force also shear forces in the spinneret and the dieswell on leaving the spinneret influence the rheological behavior of the polymer solution.

If the temperature of the water bath is higher more water vapor is present in the airgap and growth of nuclei is faster due to higher diffusion coefficients. Therefore pore sizes are expected to be larger at a higher temperature which corresponds with the experiments.

While performing more experiments than shown here it was found that the reproducibility of the spinning process with this set-up is not very good. The conditions in the airgap are susceptible to environmental changes and transport of the fiber over the rollers may easily cause an extra stretching force on the nascent fiber. To avoid these problems some modifications have been made to the spinning apparatus.

\subsection{Controlled humidity in the airgap}

The spinning set-up as depicted in Fig. 2 was used to investigate the influence of the humidity in the airgap at constant airgap length on the structure and the performance of the membranes. Three polymer solutions were used containing different amounts of PVP K90: $7.5 \mathrm{wt} \%, 10 \mathrm{wt} \%$ and $12.5 \mathrm{wt} \%$. All solutions contained $20 \mathrm{wt} \%$ PES and $5 \mathrm{wt} \%$ water. The cloud point composition of the polymer solution lies at a water content of 6-7 wt \% (extrapolated from values measured by Boom [21]). The addition of water to the polymer solution will bring its composition closer to the binodal composition. Therefore over the entire wall thickness only a small amount of water from the coagulation medium is necessary to induce demixing. The process conditions can be found in Table 2 .

In these experiments the airgap had a fixed length ( 5 $\mathrm{cm}$ ); in the airgap the humidity was varied in an independent way as described in the materials and methods section. For the three polymer casting solutions the residence time was not the same due to differences in viscosities. This resulted also in different dimensions 
Table 2

Spinning conditions and diameters of the fibers. The polymer spinning solution consisted of $20 \mathrm{wt} \%$ PES, 5 wt $\%$ water and different amounts of PVP K90 in NMP. Extrusion temperature of the solution was $60^{\circ} \mathrm{C}$ and the extrusion rate was $2.5 \mathrm{ml} / \mathrm{min}$. The bore liquid consisted of $78 \%$ $\mathrm{NMP}$ and $3 \mathrm{wt} \% \mathrm{PVP}$ in water; the temperature was $25^{\circ} \mathrm{C}$, the flow rate was $1.7 \mathrm{ml} / \mathrm{min}$ The temperature of the water bath was $20^{\circ} \mathrm{C}$

\begin{tabular}{lclll}
\hline Concentration PVP $(w t \%)$ & Viscosity $^{\mathrm{a}}$ (cpoise) & Residence time (s) & External diameter (mm) & Internal diameter (mm) \\
\hline 7.5 & 21100 & 1.0 & $1.40(5 \%)^{\mathrm{b}}$ & $0.94(5 \%)^{\mathrm{b}}$ \\
10.0 & 58600 & 1.5 & $1.55(8 \%)$ & $1.04(8 \%)$ \\
12.5 & 100800 & 2.2 & $1.94(13 \%)$ & $1.38(13 \%)$ \\
\hline
\end{tabular}

${ }^{\mathrm{a}} \mathrm{Viscosity}$ at $50^{\circ} \mathrm{C}$ extrapolated to shear force zero.

bumbers between brackets are deviations from the mean values.

of the fibers as is shown in Table 2 . Small deviations in diameters are due to temperature fluctuations (45$50^{\circ} \mathrm{C}$ ) in the airgap. For the $12.5 \mathrm{wt} \%$ PVP solution the deviation is most profound because the dependency of the viscosity on the temperature is largest for this solution.

The performance of the membranes, spun at different humidities in the airgap, is given in Fig. 4 where fluxes for water and for a BSA solution as well as the retention for BSA are plotted versus the vapor pressure of water in the airgap. The trend in the curves is the same for the three plots. The morphologies of the membranes

a)

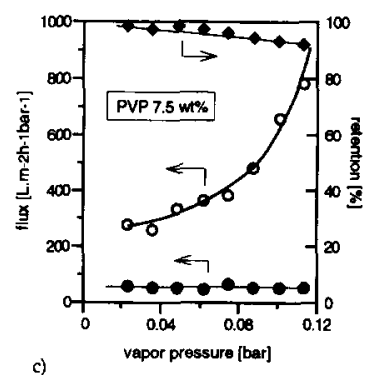

c)

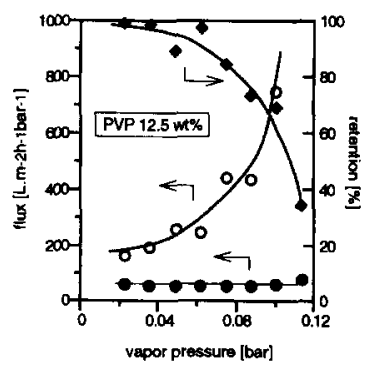

Fig. 4. Performance of the membranes spun with different vapor pressures in the airgap: pure water flux and flux and retention for a $0.1 \mathrm{wt} \%$ BSA solution. The spinning conditions were as mentioned in Table 2: (a) $7.5 \mathrm{wt} \%$ PVP, (b) $10 \mathrm{wt} \%$ PVP and (c) $12.5 \mathrm{wt} \%$ PVP in the spinning solution. Deviations from mean values were for water fluxes within $10 \%$ and for retentions within $2 \%$. are studied using SEM. In Fig. 5 the photographs of the outer surface for the solution containing $12.5 \mathrm{wt} \%$ PVP are shown.

The photographs (Fig. 5) show that pore sizes in the skin of the membranes increase when a higher water vapor pressure was used in the airgap. The same trend was found for the flux measurements. When the humidity in the airgap goes up, the water flux of the membranes increased while the retention for BSA decreased. This result is in accordance with the theory of membrane formation. More penetration of water will lead to a higher water content of the polymer solution. Upon phase separation the volume of the polymer (PES) lean phase is larger, resulting in a higher porosity of the ultimate membrane. It is clear that in this experiment for one composition of the polymer solution the gravity forces are constant.

In Fig. 4 it can be seen that at a high vapor pressure in the airgap the $12.5 \mathrm{wt} \%$ PVP solution shows a higher water flux and a lower BSA retention compared to the values of the solutions containing less PVP. According to Boom et al. $[22,23]$ the demixing gap for the short time scale is lying further to the right hand side of the phase diagram when the PVP concentration is higher. Thus at high PVP concentration more water diffuses into the polymer solution. Unlike the virtual binodal the cloud point curve is not very dependent on the PVP concentration. The phase separation process starts at the same water content but during phase separation the polymer solution with $12.5 \mathrm{wt} \%$ PVP contains more water. Also the residence time in the airgap is longer due to the high viscosity of the $12.5 \mathrm{wt} \%$ solution and water vapor has more time to diffuse into the nascent membrane. A third effect is that during phase separation the PVP also diffuses towards the polymer (PES) lean phase. More PVP (at the same PES concentration) 

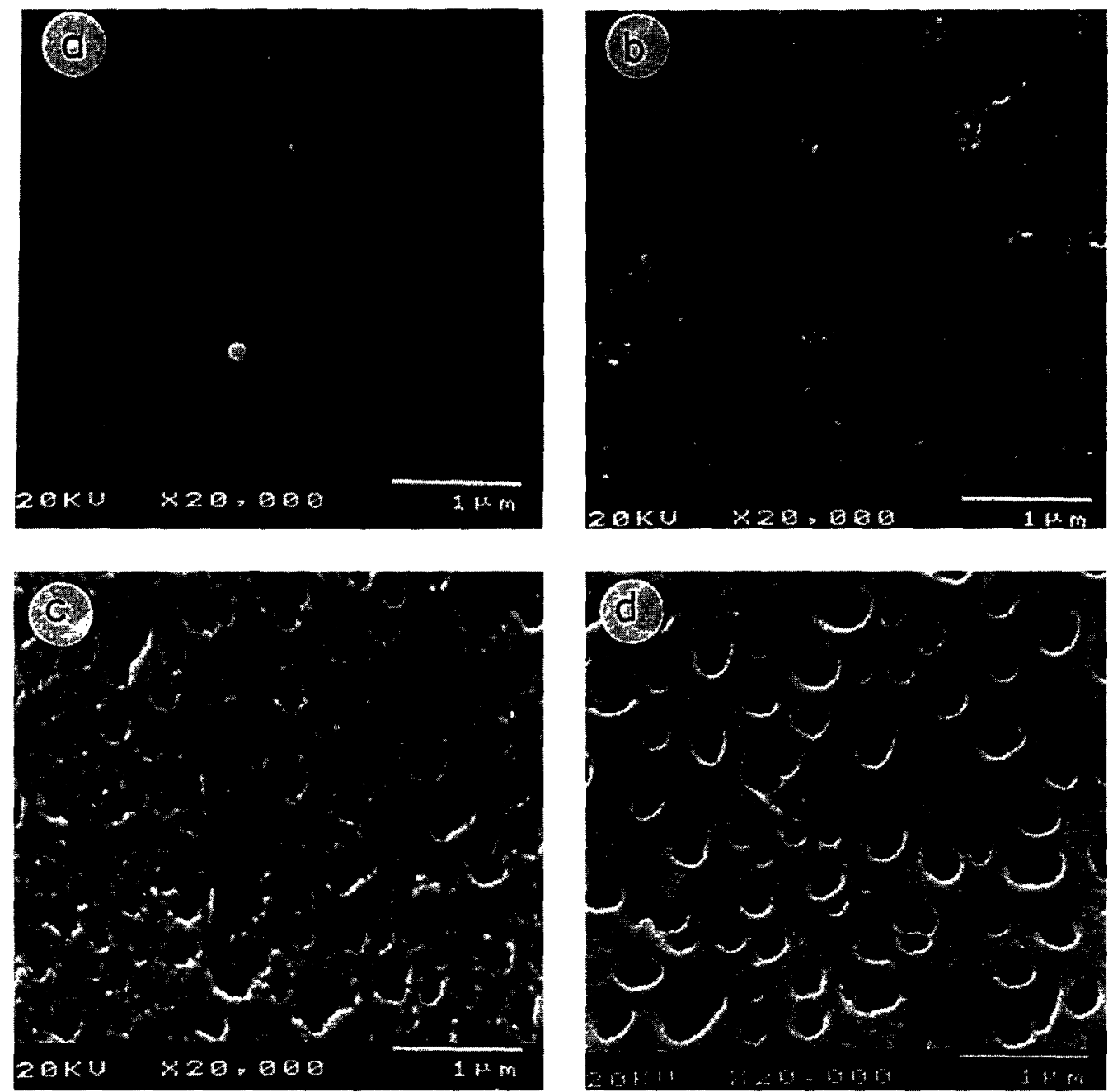

Fig. 5. Morphologies at the outer surface of the membranes, photographs made with SEM. The spinning solution contained $12.5 \mathrm{wt} \%$ PVP. Vapor pressure in the airgap was: (a) $0.036 \mathrm{bar}$, (b) 0.062 bar, (c) 0.101 bar and (d) 0.114 bar.

would thus result in a larger volume of the polymer lean phase and a larger pore volume in the ultimate membrane.

At low vapor pressure in the airgap the differences of the three polymer solutions are not so profound. Here the water flux for the $12.5 \mathrm{wt} \%$ solution is even lower than for the fibers with lower PVP content. The reason for this is that at low vapor pressure the phase separation of the polymer solution will probably not start in the airgap. In the water bath the phase separation and thus pore formation is rapidly followed by vitrification of the polymer (PES) rich phase. The vitrification point is reached sooner if the viscosity of the initial polymer concentration is higher as is the case for the $12.5 \mathrm{wt} \%$ PVP solution.

After the fiber has passed the airgap it enters into the water bath. Here the nascent membrane undergoes a temperature quench from 50 to $20^{\circ} \mathrm{C}$. Since NMP is very well miscible with water the outdiffusion of solvent is fast. In the top layer these two effects will lead to rapid vitrification of the polymer rich phase. The fast vitrification in the top layer is nicely illustrated in Fig. 6 . In the cross section the smallest pores are not found at the outer surface but about $10 \mu \mathrm{m}$ deeper into the membrane. This pore size distribution in the top layer is a direct result of the concentration profile of water that has diffused into the polymer solution in the 


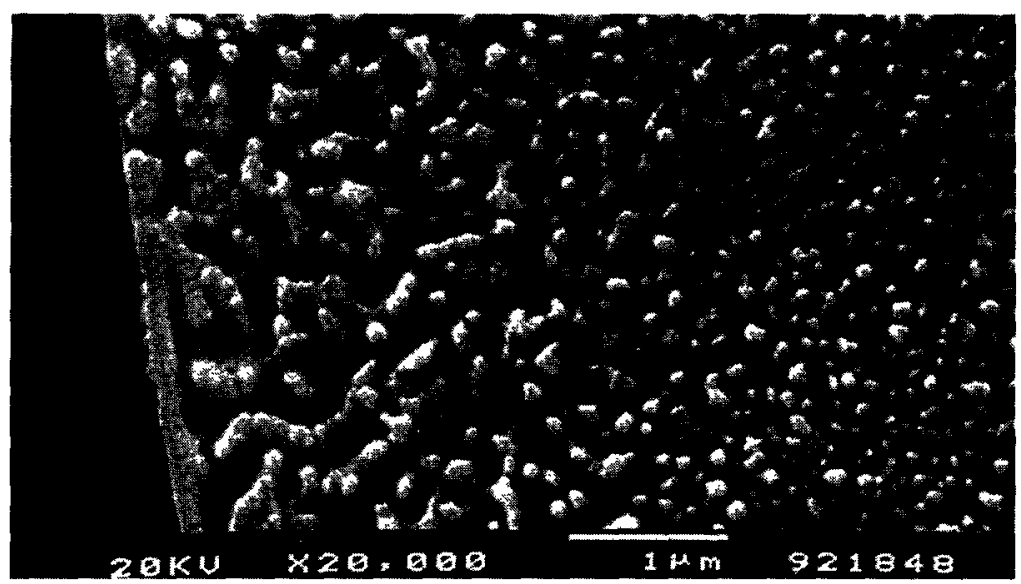

Fig. 6. Cross section of a toplayer showing the transition from the airgap into the water bath. PVP concentration in the polymer solution was 10 $\mathrm{wt} \%$, vapor pressure in the airgap was 0.114 bar. The surface pore structure (not visible on this photograph) is comparable to the one shown in Fig. 5d.

airgap. This phenomenon was also found by Roesink [5]. It can be diminished by an increase of the temperature of the water bath but this was not the aim of our research. In this study the fast coagulation in the water bath implies that the membrane structures found at the outer surface are mainly determined by the conditions in the airgap. Further away from the interface (at distances larger than $10 \mu \mathrm{m}$ ) the phase separation process is slowed down because the top layer forms a barrier for the diffusion of solvent and nonsolvent. The pore size will therefore be larger the further the pores are situated from the surface. In Fig. 6 it can be seen that the pore interconnectivity is very good which indicates that the structure is most likely formed by spinodal decomposition of the polymer solution.

Two more comments have to be made on the results presented here. The SEM photographs of Fig. 5 show that for low vapor pressures in the airgap a nodular structure in the top layer is formed. The formation mechanism of this structure is the subject of related publications [20]. The second remark concerns the flux for the BSA solution. This flux is almost the same in all cases. The flux decline from water flux to the flux for the BSA solution however is higher as the pores are larger. This is due to pore blocking of the large pores by BSA molecules [25].

\section{Conclusions}

Ultrafiltration membranes can be spun from the system PES, PVP, NMP and water. To be able to control the pore sizes at the outer surface by applying an airgap a distinction has to be made between effects caused by forces acting on the polymer spinning solution (gravity, shear forces in the spinneret, die-swell) and demixing processes. The forces on the polymer solution can be very important. Only when these have been taken into account, are results in accordance with the theory on phase separation.

\section{Acknowledgements}

The authors like to thank $\mathrm{H}$. Teunis for performing part of the spinning experiments.

\section{References}

[1] P. Aptel, N. Abidine, F. Ivaldi and J.P. Lafaille, Polysulfone hollow fibers, effect of spinning conditions on ultrafiltration properties, J. Membrane Sci., 22 (1985) 199.

[2] J.M. Espenan and P. Aptel, Outer skinned hollow fibers spinning and properties, Membranes and Membrane Processes, E. Drioli and M. Nakagaki (Eds.), Plenum, New York, 1986.

[3] I. Cabasso, E. Klein and J.K. Smith, Polysulfone hollow fibers. I. Spinning and properties, J. Appl. Polym. Sci., 20 (1976) 2377.

[4] I. Cabasso, E. Klein and J.K. Smith, Polysulfone hollow fibers. II. Morphology, J. Appl. Polym. Sci., 21 (1977) 165.

[5] H.D.W. Roesink, Microfiltration, Membrane Development and Module Design, Thesis, University of Twente, The Netherlands, 1989. 
[6] S. Doi and K. Hamanaka, Pore size control technique in the spinning of polysulfone hollow fiber ultrafiltration membranes, Desalination, 80 (1991) 167.

[7] C. Hall, Polymer materials: An Introduction for Technologists and Scientists, 2nd ed., MacMillan Education, Basingstoke, 1989. p. 42.

[8] D.R. Dreger. The polysulfones, Machine Design, 50 (1978) 114.

[9] (a) L.E.S. Brink and D.J. Romijn, Reducing the protein fouling of polysulfone surfaces and polysulfone ultrafiltration membranes: Optimization of the type of presorbed layer, Desalination, 78 ( 1990) 209; (b) G. Capannelli, A. Bottino, V. Gekas and G. Tragardh, Protein fouling behavior of ultrafiltration membranes prepared with varying degrees of hydrophilicity, Proc. Biochem. Int., December (1990) 221; (c) A.S. Jonsson and B. Jonsson, The influence of nonionic and ionic surfactants on hydrophobic and hydrophilic ultrafiltration membranes, J. Membrane Sci., 56 ( 1991 ) 49.

[10] H.D.W. Roesink, M.A.M. Beerlage, W. Potman, Th. van den Boomgaard, M.H.V. Mulder and C.A. Smolders, Characterization of new membrane materials by means of fouling experiments: Adsorption of BSA on polyetherimidepolyvinylpyrrolidone membranes, Colloids Surfaces, 55 (1991) 231

[11] L.Y. Lafreniere, F.D.F. Talbot, T. Matsuura and S. Sourirajan, Effect of polyvinylpyrrolidone additive on the performance of polyethersulfone ultrafiltration membranes, Ind. Eng. Chem. Res., 26 ( 1987$) 2385$.

[12] T.A. Tweddle, O. Kutowy, W.L. Thayer and S. Sourirajan, Polysulfone ultrafiltration membranes, Ind. Eng. Chem. Prod. Res. Dev., 22 ( 1983 ) 320.

[13] X. Xie-qing. Additive reagents for ultrafiltration membranes of polysulfone resin, Maku, 8 (1983) 311.

[14] S. Munari, A. Bottino, G. Capannelli, P. Moretti and, P. Petit Bon, Preparation and characterization of polysulfonepolyvinylpyrrolidone based membranes, Desalination, 70 (1988) 265.

[15] C. Cohen, G.B. Tanny and S. Prager, Diffusion controlled formation of porous structures in ternary polymer systems, J. Polym. Sci., Polym. Phys. Ed., 17 ( 1979$) 477$.

[16] (a) A.J. Reuvers, J.W.A. v.d. Berg and C.A. Smolders, Formation of membranes by means of immersion precipitation. I. A model to describe mass transfer during immersion precipitation, J. Membrane Sci., 34 (1987) 45; (b) A.J. Reuvers and C.A. Smolders, Formation of membranes by means of immersion precipitation. II. The mechanism of formation of membranes prepared from the system cellulose acetate-acetone-water, ibid., 34 (1987) 67.

[17] C.S. Tsay and A.J. McHugh, Mass transfer modeling of asymmetric membrane formation by phase inversion, J. Polym. Sci., Part B, Polym. Phys., 28 (1990) 1327.

[18] (a) P. Radovanovic, S.W. Thiel and S.T. Hwang, Formation of asymmetric polysulfone membranes by immersion precipitation. Part I: Modelling mass transport during gelation, J. Membrane Sci., 65 ( 1992) 213; (b) P. Radovanovic, S.W. Thiel and S.T. Hwang, Formation of asymmetric polysulfone membranes by immersion precipitation. Part II: The effects of casting solution and gelation bath compositions on membrane structure and skin formation, ibid., 65 (1992) 231.

[19] R.M. Boom, I.M. Wienk, Th. v.d. Boomgaard and C.A. Smolders, Microstructures in phase inversion membranes, part II. The role of a polymeric additive, J. Membrane Sci., 73 (1992) 277.

[20] I.M. Wienk, Th. van den Boomgaard and C.A. Smolders, The formation of nodular structures in the top layer of ultrafiltration membranes, J. Appl. Polym. Sci., 53 (1994) 1011.

[2I] (a) R.M. Boom, Th. van den Boomgaard and C.A. Smolders, Equilibrium thermodynamics of a quaternary membrane forming system with two polymers. I. Calculations, Macromolecules, 27 ( 1994 ) 2034; (b) R.M. Boom, H.W. Reinders, H.H.W. Rolevink, U. Cordilia, Th. van den Boomgaard and C.A. Smolders, Equilibrium thermodynamics of a quaternary membrane forming system with two polymers. II. Experiments, ibid.. 27 ( 1994 ) 2041.

[22] R.M. Boom, Th. van den Boomgaard and C.A. Smolders, Mass transfer and thermodynamics during immersion precipitation for a two polymer system: Evaluation with the system PESPVP-NMP-water, J. Membrane Sci., 90 (1994) 231.

[23] R.M. Boom, S. Zanic, Th. van den Boomgaard and C.A. Smolders, Membranes from PES and PVP: Membrane morphology and its relation to the formation mechanism, $\mathbf{J}$. Membrane Sci., submitted.

[24] R.M. Boom, H.H.W. Rolevink, Th. van den Boomgaard and C.A. Smolders, Membranes prepared from PES and PS: Comparison with the PES-PVP system, J. Membrane Sci., submitted.

[25] I.M. Wienk, Ultrafiltration Membranes From a Polymer Blend, Thesis University of Twente, The Netherlands, 1993.

[26] J. Gmehling. U. Onken and U. Weidlich, Vapor Liquid Equilibrium Data Collection. Vol. 1, Part 1, Dechema, Frankfurt, 1977. 